\title{
A pathway for spatial memory encoding
}

\author{
Brett M. Gibson ${ }^{1} \cdot$ Robert Mair $^{1}$ \\ Published online: 22 February 2016 \\ (C) Psychonomic Society, Inc. 2016
}

Summary The medial prefrontal cortex has been shown to play a role for rodents in successful completion of tasks that require spatial memory, but the pathways responsible for the transmission of spatial information to the $\mathrm{mPFC}$, and the nature and timing of such information, are unknown. Recently, Spellman, Rigotti, Ahmari, Fusi, Gogos, and Gordon (Nature, $522,309-314,2015$ ) addressed these questions in an eloquent and ingenious series of experiments, which we review in the broader context of the neurobiology of spatial memory.

\section{Keywords Navigation $\cdot$ Memory $\cdot$ Spatial learning $\cdot$ Working} memory

Memory has a long history of research in the brain sciences motivated by the critical role it plays in defining our past and preparing for the future. Spatial information presents a particular challenge for memory. While some spatial features of the environment change little with time, others are in constant flux. People and non-humans must constantly update their representation of the environment to know where objects were located and to plan routes. The modern neurobiological study of spatial memory can be traced to the early 1970s with the discovery by John O'Keefe and colleagues of cells in the hippocampus that respond when an animal is in a particular place in an environment. Later, in the 1980s, James Ranck identified head direction cells that are active when an animal's head is pointed in a particular direction and provide directional inputs into the place system. More recently, May-Britt Moser and Edvard Moser discovered cells in the entorhinal cortex for which the firing locations form a triangular grid, providing a coordinate system for a given environment. The importance of this collective body of work was recently recognized when the

Brett M. Gibson

bgibson@unh.edu

1 Department of Psychology, McConnell Hall, University of New Hampshire, Durham, NH 03824, USA
2014 Nobel Prize in Physiology or Medicine was awarded to O'Keefe, Moser, and Moser.

Spatially-modulated neurons play a large role in spatial working memory. Information from these cells form the framework for space that is transmitted to other structures in the brain involved in updating representations of the environment as well as performing related actives such as planning. Convergent evidence suggests that the medial prefrontal cortex (mPFC) plays a key role for rodents in the successful completion of tasks that require spatial working memory. The ventral hippocampus (vHPC) has robust projections to mPFC. While damage to this part of the hippocampus has been shown to disrupt neural activity in the mPFC during a working memory task, questions remain about the timing and transmission of spatially related information between the structures during spatial memory tasks.

Recently, Spellman, Rigotti, Ahmari, Fusi, Gogos, and Gordon (2015) started to address some of these questions in an eloquent and ingenious series of experiments. They trained mice to perform a delayed non-matching to place (DNMTP) task in a T-maze. During the sample phase a mouse departed a holding area near the distal end of a start arm and traveled to an open side arm that contained food at its end (the other arm was closed to force the mouse into the sample arm). The mouse then returned to the start position and was detained during a brief delay phase. Finally, the mouse was rereleased from the start arm and both arms at the end of the T were opened; the mouse entered the previously closed arm when making a correct choice. During a subsequent experiment the researchers subdivided each of the two distal arms to create four potential locations during the choice phase to prevent the mice from developing a solution during the sample phase. One group of mice (Arch+) were injected with a virus vector in vHPC engineered to optogenetically inhibit neurons. They then showed that optical stimulation in MPFC inhibited the terminal fields of the vHPC-mPFC pathway without affecting vHPC activity. Another group of mice (Arch-) served as a comparison and were not injected with the Archexpressing vector. Following DNMTS acquisition, the researchers delivered light to the vHPC-mPFC terminal fields during the entire trial, sample, delay, or choice phase of 
DNMTS trials. Notably, the accuracy for the goal arm dropped significantly for Arch+ mice compared to Arch- mice following light treatment but only when applied during the sample phase. Thus, inhibiting the terminal fields disrupted encoding but not maintenance or retrieval of the spatial cues necessary for successful choices during DNMTS.

These results suggest that input from the vHPC has a direct influence on the representation of spatial information in $\mathrm{mPFC}$ during the encoding phase of the DNMTS task. To examine this hypothesis Spellman et al. then went on to record from neurons in the MPFC during the four-goal version of the DNMTS task. A learning linear classifier was used to discern neural activity that predicted the sample goal location during the various phases of the trial. Sample goal location was predicted at a high level by the classifier when mice entered and were in the goal arm, but dropped during the other phases of the trial. Likewise, the classifier was highly accurate in predicting the sample goal for Arch+ animals when the light was off but plummeted when the researchers turned the light on during the sample phase of the trial. Thus, representations of the sample goal in $\mathrm{mPFC}$ appear to require input from vHPC during coding but not during other phases of the trial. The results from this experiment were particularly useful since they mirrored the drop in accuracy following inactivation reported in the first experiment.

In a final experiment, Spellman et al. examined the potential for the coordination of neural activity between the vHPC and the mPFC. Synchronous activation of populations of neurons at a common rate of oscillation is thought to create optimal conditions for neural transmission between different regions of the brain. Previous studies have demonstrated longrange gamma oscillation synchrony between hippocampus and cortex during spatial learning and memory. Spellman et al. found that the highest percentage of units that were in phase together occurred when vHPC gamma activity preceded firing in $\mathrm{mPFC}$. The researchers next inhibited vHPC terminals in $\mathrm{mPFC}$ and found a significant decrement in gamma-related synchrony. Notably, the sequence was reversed in the theta range, as $\mathrm{mPFC}$ firing preceded that in vHPC. The results support the hypothesis that vHPC influences mPFC though synchronous gamma oscillations.

These experiments are important because the results show that the vHPC input to mPFC is critical for the encoding and updating of spatial cues during a working memory task. Inhibition of vHPC-mPFC terminal fields disrupted both neural firing in the PFC and accuracy for the correct location during the working memory task. Likewise, neural activity in $\mathrm{VHPC}$ was phase locked and preceded activity in the $\mathrm{mPFC}$ for a large proportion of cells encoding the goal location. While this work has led to a number of important discoveries, additional interesting questions remain. For instance, what is the nature of the information contained in the representation? Cells that were selected for some of the analyses fired when one or more broad spatial features defined a goal location (e.g., front/back; right/left). It remains unclear what the extent of the spatial representation that may be encoded by vHPC-mPFC projections is and whether this information is based on allocentric or egocentric cues or both. Likewise, past work has indicated that neurons in the $\mathrm{MPFC}$ may hold onto representations during a delay period that could be used during later choice. That was not the case here, as the current work indicated that the vHPC-mPFC pathway was important in encoding and updating the representation of the goal environment and was not necessary during the retrieval phase of the trial. Thus, the representation supported by this pathway is called upon during the choice phase but may be maintained by other intermediate brain structures.

The mediodorsal, intralaminar, anterior, and reuniens/ rhomboid nuclei are higher-order thalamic nuclei that have substantial connections with the prefrontal cortex (PFC), hippocampus, and the basal ganglia. Until recently the thalamus was viewed primarily as a relay with minimal effects on information transmitted to the cortex. This view has changed with the recognition that the thalamus contains higher-order nuclei that are driven by cortico-thalamic and pallido-thalamic inputs, strongly influence cortical processing, and have profound effects on cognitive behavior. The thalamus may also provide rapid and precise modulation of $\mathrm{MPFC}$ - and PFCrelated pathways involving the basal ganglia and hippocampus that support spatial working memory and goal-directed behaviors. Thus, it is possible that thalamic circuits support mPFC activity during other phases of a working memory task, such as during choice or delay.

Finally, the HPC and mPFC play a broader role in attention and memory than coding space and it is unclear what contributions the vHPC-mPFC pathway may make to these processes. The authors did not find evidence that vHPC input was required for encoding non-spatial trial features, but more work could explore this possibility.

Behavioral approaches to neuroscience are increasingly viewed as outdated, whereas molecular techniques in neuroscience are viewed as the future of the field. While there is some merit to this argument, as shown by the use of the optogenetic inactivation to precisely manipulate vHPC terminals in $\mathrm{MPFC}$, we view this paper as providing a nice example of the power of combining tools of modern neuroscience with more traditional techniques of behavioral neuroscience to address fundamental questions about the nature of learning and memory.

\section{References}

Spellman, T., Rigotti, M., Ahmari, S. E., Fusi, S., Gogos, J. A., \& Gordon, J. A. (2015). Hippocampal-prefrontal inputs supports spatial encoding in workign memory. Nature, 522, 309-314. doi:10. 1038/nature14445 